SU-ITP-06-20

SLAC-PUB-11926

November 4, 2018

\title{
Exact Attractive Non-BPS STU Black Holes
}

\author{
Renata Kallosh $^{1}$, Navin Sivanandam ${ }^{1,2}$, and Masoud Soroush ${ }^{1,2}$ \\ ${ }^{1}$ Department of Physics, Stanford University, Stanford CA 94305-4060, USA \\ ${ }^{2}$ SLAC, Stanford University, Stanford CA 94309, USA \\ kallosh@stanford.edu \\ navins@stanford.edu \\ soroush@stanford.edu
}

\begin{abstract}
We develop some properties of the non-BPS attractive STU black hole. Our principle result is the construction of exact solutions for the moduli, the metric and the vectors in terms of appropriate harmonic functions. In addition, we find a spherically-symmetric attractor carrying $p^{0}$ ( $D 6$ brane) and $q_{a}$ ( $D 2$ brane) charges by solving the non-BPS attractor equation (which we present in a particularly compact form) and by minimizing an effective black hole potential. Finally, we make an argument for the existence of multicenter attractors and conjecture that if such solutions exist they may provide a resolution to the existence of apparently unstable non-BPS "attractors."
\end{abstract}




\section{Contents}

1 Introduction $\quad 3$

2 STU Black Holes and the Attractor Equation $\quad 4$

2.1 The Attractor Equation .......................... 5

2.2 Solutions to the Attractor Equation . . . . . . . . . . . . . . . . . 9

3 The Exact Non-BPS Solution $\quad 10$

3.1 Harmonic Functions and U-Duality . . . . . . . . . . . . . . . . 11

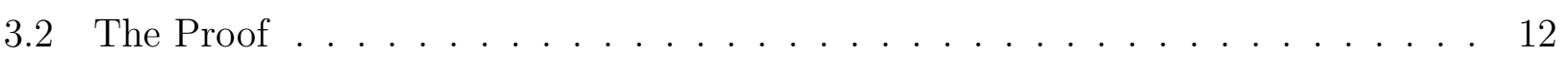

3.2 .1 The D2-D6 System . . . . . . . . . . . . . . . . . . . . . 12

3.2 .2 The Generic System: $(\mathrm{D} 0, \mathrm{D} 2, \mathrm{D} 4, \mathrm{D} 6) \ldots \ldots \ldots$

4 Split Non-BPS Attractors

$\begin{array}{llr}5 & \text { Discussion } & 17\end{array}$

A Further Details of the $D 2-D 6$ system 18

A.1 Minimizing the Effective Potential . . . . . . . . . . . . . . 18

A.2 An Alternate Attractor Equation . . . . . . . . . . . . . . . 20

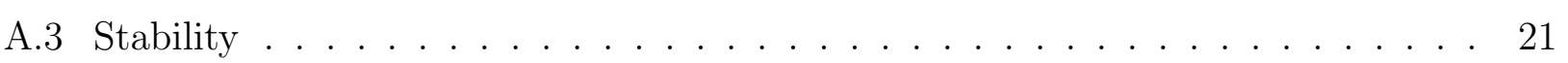




\section{Introduction}

The black hole attractor story (which began with BPS states - [1], 2], 3]) has experienced a recent flurry of activity with regards to the existence of non-BPS attractors ([4], [5]). Other interesting recent developments include the development of the attractor mechanism for flux vacua ([6], [7]), the features of $\mathcal{N}=8$ attractors $([8],[9])$ and several additional properties of both BPS and non-BPS attractors ([11], [12], [13], [10], [14] and [15]).

In particular, it seems that many of the interesting properties and features of attractive BPS configurations are shared by non-BPS ones, so long as the black hole in question remains

extremal. For example the non-BPS attractor equation was proposed in [6] and developed in [16].

Such recent developments lead one to speculate on what other attractive features may apply to non-BPS extremal black holes. However, as we try to explore this situation, an immediate problem arises. The first order BPS equations afford considerable simplifications that are not present for the more general non-BPS situation. As a result it is considerably more difficult to make progress understanding the properties of non-BPS attractors. One way to sidestep this calculational intractability is to consider a simple sub-class: The STU black holes. As outlined in more detail below, STU black holes can be constructed from compactifications of type IIA string theory whose moduli space is described by four homogeneous (or three inhomogeneous) co-ordinates. In particular, our goal in this paper is to demonstrate that, for the STU black hole, we can find exact expressions for the moduli fields, the metric and the vectors throughout the spacetime.

It is well established that, along with giving the attractive values of moduli, the BPS attractor equation can also be used to give an exact expression for the moduli fields everywhere, by taking the attractive values and replacing charges with appropriate harmonic functions (details can be found in [17], [18], [19] and [20]). While proving the equivalent statement for non-BPS black holes is difficult, it can checked explicitly in the STU case, where we will discover that the exact expressions for the moduli and the metric are given by a similar prescription as for the BPS attractors.

This paper is organized as follows. In section 2, we review the non-BPS attractor equation for STU black holes and find a new non-SUSY solution for a black hole carrying charges $\Gamma=\left(0, q_{a}, p^{0}, 0\right)$ (corresponding to wrapped $D 2$ and $D 6$ branes, and manifestly dual to the already known D0-D4 system). Next, in section 3, we perform the calculation advertised above, and confirm that the non-BPS attractor has its moduli described by appropriate combinations of harmonic functions. Finally, we speculate on the existence of multi-centered non-BPS black holes. We argue that not only are such solutions plausible, but also that they will exhibit a similar split attractor flow to the analogous BPS situation and also allow us to resolve apparently unstable "attractors" (for example, non-BPS STU extremal black holes with charges $\Gamma=$ $\left(q_{0}, q_{a}, p^{0}, p^{a}\right)$, which have fixed values of the moduli at the horizon, but are unstable to small perturbations). 


\section{STU Black Holes and the Attractor Equation}

We present here a brief review of the STU black holes and attractor behavior - further details can be found in [4], 9], [16] and [21].

When type IIA string theory is compactified on a $T^{6}$ (or, equivalently, when M-theory is compactified on $T^{7}$ ) one recovers $\mathcal{N}=8$ supergravity in $\mathrm{d}=4$ with 28 vectors and 70 scalars in the coset space $\frac{G}{H}=\frac{E_{7(7)}}{S U(8)}$, with an on-shell U-duality symmetry $E_{7(7)}$. The STU model is a consistent $\mathcal{N}=2$ truncation of a $\mathcal{N}=8$ model. The model depends on three complex moduli (parameterizing the coset space $(S L(2, \mathbb{R}) U(1))^{3}$ ) and has 4 vectors (one as an $\mathcal{N}=2$ partner of the graviton and the others in vector multiplets).

The most general one-center BPS black hole solution of an $\mathcal{N}=8$ model preserving $1 / 8$ of the available supersymmetry is a solution of the STU model, modulo U-duality [22]. To generate the most general solution one has to act with $H=S U(8)$ and transform from the $4+4$ (electric and magnetic) charges of the STU model to the $28+28$ charges of the $\mathcal{N}=8$ model. This corresponds to the decomposition of the $\mathbf{5 6}$ of $E_{7(7)}$ with respect to $S O(4,4) \times(S L(2, \mathbb{Z}))^{3}$ :

$$
56 \rightarrow\left(\mathbf{8}_{v}, \mathbf{2}, \mathbf{1}, \mathbf{1}\right) \oplus\left(\mathbf{8}_{s}, \mathbf{1}, \mathbf{2}, \mathbf{1}\right) \oplus\left(\mathbf{8}_{s^{\prime}}, \mathbf{1}, \mathbf{1}, \mathbf{2}\right) \oplus(\mathbf{1}, \mathbf{2}, \mathbf{2}, \mathbf{2}) .
$$

The charges of the STU model correspond to a singlet in $S O(4,4)$ and transform as a $(\mathbf{2}, \mathbf{2}, \mathbf{2})$ irreducible representation of $(S L(2, \mathbb{Z}))^{3}$ - shown in the last term of (2.1). Acting with $G=E_{7(7)}$ one can generate the generic values of scalars at infinity.

The bosonic part of the action of the four dimensional $\mathcal{N}=2$ supergravity (without hypermultiplets) (in units with $G_{N}=1$ ) is described by

$$
S=\frac{1}{16 \pi} \int d^{4} x \sqrt{|g|}\left(-\frac{\mathcal{R}}{2}+G_{a \bar{b}} \partial z^{a} \cdot \partial \bar{z}^{\bar{b}}+\operatorname{Im}\left(\mathcal{N}_{\Lambda \Sigma}\right) \mathcal{F}^{\Lambda} \cdot \mathcal{F}^{\Sigma}+\operatorname{Re}\left(\mathcal{N}_{\Lambda \Sigma}\right) \mathcal{F}^{\Lambda} \cdot(* \mathcal{F})^{\Sigma}\right)
$$

The fermionic part (the gravitino and the chiral gaugino) can be constructed via the supersymmetry transformations:

$$
\begin{aligned}
& \delta \psi_{A \mu}=D_{\mu} \epsilon_{A}+\varepsilon_{A B} T_{\mu \nu}^{-} \gamma^{\nu} \epsilon^{B}, \\
& \delta \lambda^{a A}=i \gamma \cdot \partial z^{a} \epsilon^{A}+\frac{i}{2} \varepsilon_{A B} \mathcal{F}^{-a} \cdot \gamma \epsilon^{B} .
\end{aligned}
$$

$\epsilon_{A}$ is the fermionic parameter of the transformation and $\varepsilon_{A B}$ is the $S O(2)$ Ricci tensor. In above transformation laws, $T^{-}$and $\mathcal{F}^{-a}$ are the two-form graviphoton and vetor multiplet field strengths respectively. The integrals of these two two-forms on some two cycles give us the central charge of the supersymmetry algebra and its moduli space covariant derivative:

$$
Z=-\frac{1}{2} \int_{S^{2}} T^{-}, \bar{D}_{\bar{a}} \bar{Z}=-\frac{1}{2} \int_{S^{2}} \mathcal{F}^{-b} G_{b \bar{a}} .
$$

The STU models correspond to the subset of the above theories with 3 vector multiplets. The moduli space of the theory is described by the four homogenous coordinates $X^{\Lambda}$. These 
coordinates combine to give the STU prepotential 11:

$$
F(X)=\frac{X^{1} X^{2} X^{3}}{X^{0}}
$$

The Kähler potential and superpotential of the theory can be readily constructed from (2.6). Working with the inhomogeneous coordinates $z^{\Lambda}=\frac{X^{\Lambda}}{X^{0}}=\left(1, z^{a}\right)(a \in\{1,2,3\})$ the Kähler potential is:

$$
K=-\ln \left(-i\left(z^{1}-\bar{z}^{1}\right)\left(z^{2}-\bar{z}^{2}\right)\left(z^{3}-\bar{z}^{3}\right)\right) .
$$

The metric and connection on the moduli space then follow immediately:

$$
G_{a \bar{b}}=-\frac{\delta_{a b}}{\left(z^{a}-\bar{z}^{a}\right)^{2}}, G^{a \bar{b}}=-\delta^{a b}\left(z^{a}-\bar{z}^{a}\right)^{2}, \Gamma_{a a}^{a}=-\frac{2}{z^{a}-\bar{z}^{a}}(\text { no sum on } a) .
$$

Let's initially assume a static, spherically-symmetric spacetime, with a metric of the form:

$$
d s^{2}=-e^{2 U(r)} d t^{2}+e^{-2 U(r)} d x^{i} d x^{i}
$$

In this background it is straightforward to define electric and magnetic charges (details can be found in appendix A of [16]) which give rise to a superpotential of the form

$$
W=q_{\Lambda} X^{\Lambda}-p^{\Lambda} \partial_{\Lambda} F,
$$

where $q_{\Lambda}$ and $p^{\Lambda}$ are electric and magnetic charges respectively. The set of charges $\left(q_{0}, q_{a}, p^{0}, p^{a}\right)$ corresponds to the charges of $(D 0, D 2, D 6, D 4)$ branes wrapped on $(0,2,6,4)$ cycles.

An effective potential for the moduli (in this spherically-symmetric, static spacetime )is given by:

$$
V_{B H}=|D Z|^{2}+|Z|^{2}=G^{a \bar{b}}\left(D_{a} Z\right) \bar{D}_{\bar{b}} \bar{Z}+Z \bar{Z} .
$$

$Z$ the central charge of the supersymmetry algebra and, in our case, is given by $Z=e^{K / 2} W$. If the black hole in this solution is extremal (i.e. has zero temperature) then it will be an attractor for the moduli - the values of the $z^{a}$ will be fixed at the horizon, independently of their values at infinity ([4], [16]).

The horizon values of the moduli can be obtained by minimizing the above effective potential - either directly or through the attractor equation described below. Note, however, that the extremal point of $V_{B H}$ must be a minimum, else the attractor will be unstable to, say, perturbations away from staticity. For BPS attractors this condition is always satisfied, for non-BPS ones, however, we have to check the second derivatives of $V_{B H}$ explicitly.

\subsection{The Attractor Equation}

In this section, we derive an identity using the symplectic properties of $\mathcal{N}=2$ supergravity. Then, by imposing the minimization condition of the black hole potential on the identity we construct an algebraic relationship between the charges and the attractive values of the moduli.

\footnotetext{
${ }^{1}$ When string theory is compactified on some spaces which break supersymmetry down to $\mathcal{N}=2$, e. g. heterotic theory on $K 3 \times T^{2}$, the prepotential (2.6) has corrections. However, when considered as a consistent truncation of $\mathcal{N}=8$ model, the prepotential is given just by eq. (2.6) .
} 
If we define the covariantly holomorphic period vector $\Pi=\left(L^{\Lambda} M_{\Lambda}\right)$, then the symplectic structure of $\mathcal{N}=2$ supergravity relates the upper and lower components of the period vector via the vector coupling in the following way:

$$
M_{\Lambda}=\mathcal{N}_{\Lambda \Sigma} L^{\Sigma}, D_{a} M_{\Lambda}=\overline{\mathcal{N}}_{\Lambda \Sigma} D_{a} L^{\Sigma}
$$

where $\Pi=\left(L^{\Lambda} M_{\Lambda}\right)=e^{\frac{K}{2}}\left(X^{\Lambda} F_{\Lambda}\right)$, and $\left(X^{\Lambda} F_{\Lambda}\right)$ is the holomorphic period vector. Now, we form a matrix by constructing the tensor product of the period vector covariant derivative with its complex conjugate

$$
\begin{aligned}
G^{a \bar{b}} D_{a} \Pi \otimes \bar{D}_{\bar{b}} \bar{\Pi} & =G^{a \bar{b}}\left(\begin{array}{cc}
D_{a} L^{\Lambda} \bar{D}_{\bar{b}} \bar{L}^{\Sigma} & D_{a} M_{\Lambda} \bar{D}_{\bar{b}} \bar{L}^{\Sigma} \\
D_{a} L^{\Lambda} \bar{D}_{\bar{b}} \bar{M}_{\Sigma} & D_{a} M_{\Lambda} \bar{D}_{\bar{b}} \bar{M}_{\Sigma}
\end{array}\right) \\
& =G^{a \bar{b}}\left(\begin{array}{cc}
D_{a} L^{\Lambda} \bar{D}_{\bar{b}} \bar{L}^{\Sigma} & \overline{\mathcal{N}}_{\Lambda \Delta} D_{a} L^{\Delta} \bar{D}_{\bar{b}} \bar{L}^{\Sigma} \\
\mathcal{N}_{\Sigma \Delta} D_{a} L^{\Lambda} \bar{D}_{\bar{b}} \bar{L}^{\Delta} & \overline{\mathcal{N}}_{\Lambda \Delta} \mathcal{N}_{\Sigma \Gamma} D_{a} L^{\Delta} \bar{D}_{\bar{b}} \bar{L}^{\Gamma}
\end{array}\right),
\end{aligned}
$$

where in the second line we have used (2.12). If we use the following special geometry identity 2

$$
G^{a \bar{b}} D_{a} L^{\Lambda} \bar{D}_{\bar{b}} \bar{L}^{\Sigma}=-\frac{1}{2} \operatorname{Im}\left(\mathcal{N}^{-1}\right)^{\Lambda \Sigma}-\bar{L}^{\Lambda} L^{\Sigma}
$$

then we find another useful identity

$$
G^{a \bar{b}} D_{a} \Pi \otimes \bar{D}_{\bar{b}} \bar{\Pi}=-\frac{i}{2}\left(\begin{array}{cc}
0 & 1 \\
-1 & 0
\end{array}\right)-\frac{1}{2} \mathcal{M}-\bar{\Pi} \otimes \Pi,
$$

where matrix $\mathcal{M}$ is defined as

$$
\mathcal{M} \equiv\left(\begin{array}{cc}
\operatorname{Im}\left(\mathcal{N}^{-1}\right)^{\Lambda \Sigma} & \operatorname{Re}(\mathcal{N})_{\Lambda \Delta} \operatorname{Im}\left(\mathcal{N}^{-1}\right)^{\Delta \Sigma} \\
\operatorname{Im}\left(\mathcal{N}^{-1}\right)^{\Lambda \Delta} \operatorname{Re}(\mathcal{N})_{\Delta \Sigma} & \operatorname{Im}(\mathcal{N})_{\Lambda \Sigma}+\operatorname{Re}(\mathcal{N})_{\Lambda \Delta} \operatorname{Im}\left(\mathcal{N}^{-1}\right)^{\Delta \Gamma} \operatorname{Re}(\mathcal{N})_{\Gamma \Sigma}
\end{array}\right)
$$

In fact, (2.15) expresses the tensor product of the covariant derivative of the period vector with its complex conjugate in terms of the tensor product of the period vector itself with its conjugate via the vector couplings.

Now, assume that $\Gamma$ is the set of magnetic and electric charges $\Gamma=\left(p^{\Lambda} q_{\Lambda}\right)$. We define $\tilde{\Gamma}$ by a symplectic rotation:

$$
\tilde{\Gamma}=\left(\begin{array}{cc}
0 & \mathbf{1} \\
-\mathbf{1} & 0
\end{array}\right) \Gamma,\left(\begin{array}{cc}
0 & \mathbf{1} \\
-\mathbf{1} & 0
\end{array}\right) \in S p\left(2\left(h^{1,1}+1\right), \mathbb{Z}\right) .
$$

Recalling (2.10), it can easily be seen that the central charge can be expressed as $Z=\tilde{\Gamma}^{t} \cdot \Pi$. Using this form, we can compute $G^{a \bar{b}}\left(D_{a} Z\right) \bar{D}_{\bar{b}} \bar{\Pi}$ using (2.15):

$$
G^{a \bar{b}}\left(D_{a} Z\right) \bar{D}_{\bar{b}} \bar{\Pi}=\tilde{\Gamma}^{t} \cdot\left(G^{a \bar{b}} D_{a} \Pi \otimes \bar{D}_{\bar{b}} \bar{\Pi}\right) .
$$

Thus, we have the following expression:

$$
2 i \bar{Z} \Pi+2 i G^{a \bar{b}}\left(D_{a} Z\right) \bar{D}_{\bar{b}} \bar{\Pi}=\Gamma-i \tilde{\Gamma}^{t} \cdot \mathcal{M} .
$$

\footnotetext{
${ }^{2}$ This identity can be proved by considering the inner product $\left\langle D_{a} \Pi, \bar{D}_{\bar{b}} \bar{\Pi}\right\rangle=-i G_{a \bar{b}}$ and using (2.12).
} 
It has been shown in [16], [23], and [24], that the symplectic invariant $I_{1}$ is given by:

$$
I_{1}=|Z|^{2}+|D Z|^{2}=-\frac{1}{2} \tilde{\Gamma}^{t} \cdot \mathcal{M} \cdot \tilde{\Gamma} .
$$

Finally, we differentiate the above expression with respect to the charges and substitute for (2.19):

$$
\Gamma+i \frac{\partial I_{1}}{\partial \tilde{\Gamma}}=2 i \bar{Z} \Pi+2 i G^{a \bar{b}}\left(D_{a} Z\right) \bar{D}_{\bar{b}} \bar{\Pi}
$$

So far, everything we have said is generically true and independent of the detailed model. However, if we restrict ourselves to STU models, then the symplectic invariant $I_{1}$ is a function of charges. It is given by $I_{1}=\sqrt{|\mathcal{W}(\Gamma)|}$, in which $\mathcal{W}$ is given by:

$$
\mathcal{W}(\Gamma)=4\left(\left(p^{1} q_{1}\right)\left(p^{2} q_{2}\right)+\left(p^{1} q_{1}\right)\left(p^{3} q_{3}\right)+\left(p^{2} q_{2}\right)\left(p^{3} q_{3}\right)\right)-\left(p^{\Lambda} q_{\Lambda}\right)^{2}-4 p^{0} q_{1} q_{2} q_{3}+4 q_{0} p^{1} p^{2} p^{3} .
$$

In [17], Berhrndt et al. demonstrated that $\mathcal{W}(\Gamma)$ is an $[S L(2, \mathbb{Z})]^{3}$ invariant which uniquely determines the form of the STU metric and further $-\mathcal{W}$ has been established as the Cayley hyperdeterminant by Duff in [25]. The fact we must use the absolute value of $\mathcal{W}$ in order that this expression be valid for non-BPS attractors was discussed in [13] - here it was observed that $\mathcal{W}(\Gamma)$ is positive for BPS attractors and negative for non-BPS ones.

Recalling that at an attractor point the effective black hole potential will be minimized (see [23], [4], 16]) we note that extremization of (2.11) gives:

$$
2 \bar{Z} D_{\hat{a}} Z+i C_{\hat{a} \hat{b} \hat{c}} \eta^{\hat{b} \hat{\bar{d}}} \eta^{\hat{c} \hat{e}}\left(\bar{D}_{\hat{\bar{d}}} \bar{Z}\right)\left(\bar{D}_{\hat{\bar{e}}} \bar{Z}\right)=0
$$

Hatted indices are for the tangent space and $\eta^{\hat{a} \hat{\bar{b}}}$ is a flat Euclidean metric. Here $G^{a \bar{a}}=e_{\hat{a}}^{a} \eta^{\hat{a} \hat{a}} e_{\hat{\bar{a}}}^{\bar{a}}$. The rank 3 completely symmetric tensor $C_{\hat{a} \hat{b} \hat{c}}$ in the tangent space is related to the covariantly holomorphic symmetric curved space symmetric tensor $C_{a b c}$ as follows: $C_{\hat{a} \hat{b} \hat{c}}=e_{\hat{a}}^{a} e_{\hat{b}}^{b} e_{\hat{c}}^{c} C_{a b c}$. Here the rank 3 completely symmetric covariantly holomorphic tensor of the special Kähler geometry, $C_{a b c}$, is defined as follows [24]:

$$
C_{a b c}=e^{K}\left(\partial_{a} X^{A} \partial_{b} X^{B} \partial_{c} X^{C}\right) \partial_{A} \partial_{B} \partial_{C} F
$$

For the STU model with the prepotential (2.6) the non-vanishing components of the symmetric 3 -tensor are $C_{\hat{1} \hat{2} \hat{3}}=1$

Note that the covariantly holomorphic symmetric 3-tensor $C_{a b c}$ has a chiral weight 2 under Kähler transformations whereas $C_{\hat{a} \hat{b} \hat{c}}$ in the tangent space has a chiral weight 0 .

The chiral $U(1)$ weights of various objects living on Kähler manifolds are defined as follows. The Kähler potential transforms as:

$$
K \rightarrow K+f(z)+\bar{f}(\bar{z}) .
$$

The covariant derivative of an object with the chiral weight $p$ is given by $D \Phi=(d+i p \mathcal{Q}) \Phi$ where the Kähler connection is given by $\mathcal{Q}=-\frac{i}{2}\left(\partial_{a} K d z^{a}-\partial_{\bar{a}} K d \bar{z}^{\bar{a}}\right)$. In components this means:

$$
D_{a} \Phi=\left(\partial_{a}+\frac{1}{2} p \partial_{a} K\right) \Phi .
$$


Thus the chiral weight of $Z$ is $1, \bar{Z}$ is $-1, e_{\hat{a}}^{a}$ is $-2 / 3$ and $e_{\hat{a}}^{\bar{a}}$ is $2 / 3$. This means, in particular, that under Kähler transformations the central charge $Z$ as well as its covariant derivative transform by a phase:

$$
Z \rightarrow Z e^{\frac{\bar{f}-f}{2}}, \quad D_{\hat{a}} Z \rightarrow D_{\hat{a}} Z e^{\frac{\bar{f}-f}{6}} .
$$

Further, observe that (2.23) has a chiral weight $-2 / 3$ whereas (2.21) has a vanishing chiral weight. In [9] (2.23) was studied by embedding the $\mathcal{N}=2$ supergravity into an $\mathcal{N}=8$ one. Following the same method we define $Y_{0} \equiv i Z$, and $Y_{a} \equiv \bar{D}_{\hat{a}} \bar{Z}$. This gives us the solution:

$$
Y_{0}=\rho e^{i(\pi-3 \phi)}, Y_{a}=\rho e^{i \phi} .
$$

Here the arbitrary phase $\phi$ reflects the possibility to perform a Kähler transformations in agreement with the weights shown in (2.27). (2.21) can be rewritten in the following form:

$$
\Gamma+i \frac{\partial I_{1}}{\partial \tilde{\Gamma}}=2 \rho\left(\Pi+i \sum_{\hat{a}=1}^{3} \bar{D}_{\hat{a}} \bar{\Pi}\right)
$$

The arbitrary phase $\phi$ drops from this equation since the left hand side of eq. (2.21) is manifestly Kähler invariant and in the right hand side the phase cancels in each term separately.

Now, if we divide (2.29) by its zeroth component, then we follow 9 and obtain:

$$
\begin{aligned}
& \frac{p^{\Lambda}+i \frac{\partial I_{1}}{\partial q_{\Lambda}}}{p^{0}+i \frac{\partial I_{1}}{\partial q_{0}}}=\frac{L^{\Lambda}+i \sum_{\hat{a}=1}^{3} \bar{D}_{\hat{a}} \bar{L}^{\Lambda}}{L^{0}+i \sum_{\hat{a}=1}^{3} \bar{D}_{\hat{a}} \bar{L}^{0}} \\
& \frac{q_{\Lambda}-i \frac{\partial I_{1}}{\partial p^{\Lambda}}}{q_{0}-i \frac{\partial I_{1}}{\partial p^{0}}}=\frac{M_{\Lambda}+i \sum_{\hat{a}=1}^{3} \bar{D}_{\hat{\widehat{a}}} \bar{M}_{\Lambda}}{M_{0}+i \sum_{\hat{a}=1}^{3} \bar{D}_{\hat{a}} \bar{M}_{0}} .
\end{aligned}
$$

In fact, these equations are dual to each other and either set is sufficient to completely determine the moduli at the horizon.

We now illustrate that an even simpler form of these equations can be found, as the r.h.s simplifies significantly. Consider (2.30), since (with the vielbein $e_{\hat{b}}^{a}$ given by $e_{\hat{b}}^{a}=i \delta_{b}^{a}\left(z^{a}-\bar{z}^{a}\right)$ ):

$$
D_{\hat{b}} L^{c}=e_{\hat{b}}^{a} D_{a} L^{c}=e^{K / 2} e_{\hat{b}}^{a}\left(\delta_{a}^{c}+\left(\partial_{a} K\right) z^{c}\right),
$$

we can readily establish:

$$
L^{c}+i \sum_{\hat{a}=1}^{3} \bar{D}_{\hat{a}} \bar{L}^{c}=-2 e^{K / 2} \bar{z}^{c}, L^{0}+i \sum_{\hat{a}=1}^{3} \bar{D}_{\hat{a}} \bar{L}^{0}=-2 e^{K / 2} .
$$

As expected the above is independent of charges, and the values of the moduli at the horizon for any $\Gamma$ are thus given by:

$$
z^{\Lambda}(\Gamma)=\frac{p^{\Lambda}-i \frac{\partial I_{1}(\Gamma)}{\partial q_{\Lambda}}}{p^{0}-i \frac{\partial I_{1}(\Gamma)}{\partial q_{0}}} .
$$

This appears to be the simplest form of the non-BPS attractor equation for the STU model. In order to obtain eq. (2.34), we have used the gauge in which $X^{0}$ was real. However, the 
attractor equation can be generalized 3 to the case in which $X^{0}=e^{-\frac{3 i \delta}{4}}$, with real $\delta$. In this gauge the attractor equation generalizing the one in eq. (2.34) becomes

$$
\frac{z^{c}-e^{i \delta}\left(2 \bar{z}^{c}+z^{c}\right)}{1-3 e^{i \delta}}=\frac{p^{c}+i \frac{\partial I_{1}(\Gamma)}{\partial q_{c}}}{p^{0}+i \frac{\partial I_{1}(\Gamma)}{\partial q_{0}}}
$$

and similar equation for component $M_{\Lambda}$. In case that $\delta=2 n \pi$ eq. (2.35) reduces to eq. (2.34). This gives a correct solution for (D2-D6) and (D0-D4) systems. However, if one considers (D0-D6) system, then eq. (2.35) requires $\delta=(2 n+1) \pi$.

For the BPS case $Y_{a}$ vanishes and so a similar procedure to that used above will result in an attractor equation of the form of (2.29), but with the r.h.s. containing only the first term. Dividing such an expression by its zeroth component we would obtain:

$$
z^{\Lambda}(\Gamma)=\frac{p^{\Lambda}+i \frac{\partial I_{1}(\Gamma)}{\partial q_{\Lambda}}}{p^{0}+i \frac{\partial I_{1}(\Gamma)}{\partial q_{0}}}
$$

Of course, this is not simply the complex conjugate of (2.34), as in the BPS case $\mathcal{W}>0$, whilst for the non-BPS case $\mathcal{W}<0$ (recall $\left.I_{1}=\sqrt{|\mathcal{W}|}\right)$.

\subsection{Solutions to the Attractor Equation}

It is relatively straightforward to obtain solutions to the above attractor equation when we restrict ourselves to a system with only $D 0$ and $D 4$ branes ( $q_{0}$ and $p^{a}$ charges). This attractor was found in [5] by minimizing $V_{B H}$ - in fact, the solution here is not restricted to STU, i.e. there may be any number of the moduli. We consider here, though, the complementary solution with $D 6$ and $D 2$ branes ( $p^{0}$ and $q_{a}$ charges). The superpotential is then given by:

$$
W\left(z^{1}, z^{2}, z^{3}\right)=q_{a} z^{a}+p^{0} z^{1} z^{2} z^{3}
$$

It is also straightforward to write down the symplectic invariant $I_{1}$ :

$$
I_{1}=\sqrt{ \pm 4 p^{0} q_{1} q_{2} q_{3}}
$$

A positive sign under the square root corresponds to the non-BPS attractor and a negative one to the BPS one, since, as discussed above, $\mathcal{W}(\Gamma)$ is generally positive for charges corresponding to BPS solutions and negative for those corresponding to non-BPS ones (it is evident from the form of the solutions below that $p^{0} q_{1} q_{2} q_{3}<0$ for BPS attractors $p^{0} q_{1} q_{2} q_{3}>0$ otherwise).

¿From (2.38) it is trivial to use (2.36) to obtain the BPS attractive moduli values as:

$$
z^{1}=-i \sqrt{-\frac{q_{2} q_{3}}{p^{0} q_{1}}}, z^{2}=-i \sqrt{-\frac{q_{1} q_{3}}{p^{0} q_{2}}}, z^{3}=-i \sqrt{-\frac{q_{1} q_{2}}{p^{0} q_{3}}} .
$$

\footnotetext{
${ }^{3}$ We are grateful to E. Gimon who suggested to look for a more general form of eq. (2.34) which would include (D0-D6) system.
} 
The non-BPS (from (2.34)) results have a similar form:

$$
z^{1}=-i \sqrt{\frac{q_{2} q_{3}}{p^{0} q_{1}}}, z^{2}=-i \sqrt{\frac{q_{1} q_{3}}{p^{0} q_{2}}}, z^{3}=-i \sqrt{\frac{q_{1} q_{2}}{p^{0} q_{3}}} .
$$

These results have been confirmed by minimizing $V_{B H}$ and by using the form of the attractor equation given in [16] - these details of those calculations can be found in appendices A.1 and A.2. It's worth noting at this juncture that care must be taken with these solutions to ensure that their signs are such that $e^{K}$ is positive. This point is discussed in somewhat more detail in the appendix. We also observe that (2.34) and (2.36) can be used to find the attractor values of the moduli for the D0-D4 system addressed in [5] and [16]. As expected the BPS answers are:

$$
z^{1}=-i \sqrt{\frac{q_{0} p^{1}}{p^{2} p^{3}}}, z^{2}=-i \sqrt{\frac{q_{0} p^{2}}{p^{3} p^{1}}}, z^{3}=-i \sqrt{\frac{q_{0} p^{3}}{p^{1} p^{2}}} .
$$

And the non-BPS:

$$
z^{1}=-i \sqrt{-\frac{q_{0} p^{1}}{p^{2} p^{3}}}, z^{2}=-i \sqrt{-\frac{q_{0} p^{2}}{p^{3} p^{1}}}, z^{3}=-i \sqrt{-\frac{q_{0} p^{3}}{p^{1} p^{2}}} .
$$

As mentioned above, for a non-BPS attractor we are required to confirm that the extremal point of the potential does correspond to a minima. It turns out that this is a somewhat subtle and involved calculation. The details can be found in appendix A.3, but the short-answer to the question of stability is (as in the D0-D4 case discussed in [5]): "It's stable."

Before moving on to a discussion of the complete solutions for the moduli fields we summarize our knowledge of STU black hole attractors:

- All extremal STU black holes can exhibit attractor behavior, with the values of the moduli at the horizon found by extremizing the effective potential $V_{B H}$.

- Only those systems with extremum of the potential a minimum will form stable attractors. These systems include those with $\Gamma=\left(q_{0}, 0,0, p^{a}\right)$ and those with $\Gamma=\left(0, q_{a}, p^{0}, 0\right)$. Unstable "attractors" include those with all four types of charge, $\Gamma=\left(q_{0}, q_{a}, p^{0}, p^{a}\right)$; this result was established in [5] (see appendix A.3 for details).

We shall return to the issue of stability when we consider multi-centered non-BPS black holes in section 4. Now, however, we move on to the general solution for the moduli.

\section{The Exact Non-BPS Solution}

We now demonstrate explicitly the construction of exact solutions for the fields in the non-BPS attractor. We begin with a general discussion, and then write down and prove the form of the solutions. 


\subsection{Harmonic Functions and U-Duality}

So far, we have calculated the value of moduli at the horizon of the supersymmetric and nonsupersymmetric STU black holes via the attractor mechanism. It turns out, however, that we can do better than that - we can find solutions to the equations of motion for the moduli, allowing us to obtain their values everywhere. This has already been done for the BPS case (details can be found in [17], [18], [19] and [27]), and we will proceed in an analogous fashion.

We work with the single-center spherically-symmetric, static metric ansatz:

$$
d s^{2}=-e^{2 U} d t^{2}+e^{-2 U} d x^{i} d x^{i} .
$$

The basic idea is that one takes the horizon $\left(r=r_{h}\right)$ values of the moduli, $z^{a}\left(q_{\Lambda}, p^{\Lambda}\right)$ and replaces the charges with harmonic functions:

$$
z^{\Lambda}(H(\mathbf{x}))=\frac{H^{\Lambda}-i \frac{\partial I_{1}(H)}{\partial H_{\Lambda}}}{H^{0}-i \frac{\partial I_{1}(H)}{\partial H_{0}}}
$$

The metric is found through:

$$
e^{-2 U}=I_{1}(\mathbf{H})
$$

The harmonic functions $\mathbf{H}$ are:

$$
\mathbf{H}(\tau)=\left(H^{\Lambda}, H_{\Lambda}\right)=\left(\Gamma^{\Lambda}, \Gamma_{\Lambda}\right) \tau+\left(h^{\Lambda}, h_{\Lambda}\right), \tau=\frac{1}{\left|r-r_{h}\right|} .
$$

$I_{1}$ is the symplectic invariant defined above and there is a constraint on $\mathbf{h}:\langle\mathbf{h}, \boldsymbol{\Gamma}\rangle=0$. $\mathbf{H}$ arises from the solution to the vector equations of motion where the vector fields can be represented by the doublet $\left(F^{\Lambda}, G_{\Lambda}\right)$. These fields are not independent:

$$
G_{\Lambda}=\operatorname{Re} \mathcal{N}_{\Lambda \Sigma}(z, \bar{z}) F^{\Lambda}-\operatorname{Im} \mathcal{N}_{\Lambda \Sigma}(z, \bar{z}) * F^{\Lambda}
$$

The equations of motion then require a vector field potential given by $\left(\mathcal{A}^{\Lambda}, \mathcal{B}_{\Lambda}\right)$ where $F^{\Lambda}=d \mathcal{A}^{\Lambda}$ and $G_{\Lambda}=d \mathcal{B}_{\Lambda}$. The vector fields are related to the harmonic functions by:

$$
F_{m n}^{\Lambda}=\frac{1}{2} \epsilon_{m n p} \partial_{p} H^{\Lambda}, G_{\Lambda m n}=\frac{1}{2} \epsilon_{m n p} \partial_{p} H_{\Lambda} .
$$

The timelike components of $F$ and $G$ are fixed in terms of the spatial ones through (3.5).

That this result holds in the BPS case has been established explicitly; there is, however, no such proof for non-BPS black holes. Instead, we shall apply the above algorithm to generate an ansatz which can be checked explicitly using the STU equations of motion. Before we do so, though, we offer an argument as to why one might expect the above prescription to work.

There exists a manifest $[S L(2, \mathbb{Z})]^{3}$ symmetry of the equations of motion which mixes the Maxwell equations and Bianchi identities:

$$
\left(\begin{array}{c}
\mathcal{A}^{\Lambda}(\vec{x}) \\
\mathcal{B}_{\Lambda}(\vec{x})
\end{array}\right)^{\prime}=\left(\begin{array}{cc}
A & B \\
C & D
\end{array}\right)\left(\begin{array}{c}
\mathcal{A}^{\Lambda}(\vec{x}) \\
\mathcal{B}_{\Lambda}(\vec{x})
\end{array}\right)
$$


Here $\left(\begin{array}{cc}A & B \\ C & D\end{array}\right)$ is a global $S p(8, \mathbb{Z})$ matrix such that $A^{T} C-C^{T} A=B^{T} D-D^{T} B=0$ and $A^{T} D-C^{T} B=1$. Only a subgroup of this symmetry, corresponding to $[S L(2, \mathbb{Z})]^{3}$ duality symmetry, is unbroken at the level of solutions to the equations of motion. The details of the embedding of $[S L(2, \mathbb{Z})]^{3}$ into $S p(8, \mathbb{Z})$ are given in [21] and [26]. Simultaneously with the duality transformations of the vector potential doublet the 3 moduli also undergo a standard fractional transformation of the type:

$$
\left(z^{i}(\vec{x})\right)^{\prime}=\frac{a z^{i}(\vec{x})+b}{c z^{i}(\vec{x})+d} .
$$

This transformation of the moduli follows from the consistency of equation (3.5) and leaves the metric invariant

$$
\left(g_{\mu \nu}(\vec{x})\right)^{\prime}=g_{\mu \nu}(\vec{x}),
$$

allowing us to generate from one solution all the others related through duality. Clearly, such a symmetry places strong constraints on the form of the moduli fields. Further, our explorations of functional forms have been unable to find any solutions with the appropriate symmetry other than those given by (3.2), when we simultaneously impose the obvious criteria that the moduli give the correct horizon values, remain bounded everywhere and have derivatives that do the same while also vanishing at the horizon (see [16] for the origins of these restrictions).

\subsection{The Proof}

We shall begin by establishing that the appropriate ansatz solves the $D 2-D 6$ brane system and then find the general (almost - see below for details) solution through a symmetry argument.

\subsubsection{The $D 2-D 6$ System}

The four dimensional stationary, spherically-symmetric effective Lagrangian of the MaxwellEinstein action (derived from (2.2) ) is:

$$
\mathcal{L}\left(U(\tau), z^{a}(\tau), \bar{z}^{\bar{a}}(\tau)\right)=\left(\dot{U}^{2}+G_{a \bar{b}} \dot{z}^{a} \dot{\bar{z}}^{\bar{b}}+e^{2 U} V_{B H}\right)
$$

$\tau$ is the inverse of the radial coordinate (such that the horizon is at $\tau=-\infty$ and the derivatives denote differentiation with respect to $\tau$. The gravitational and scalar equations of motion derived from the above Lagrangian (3.10) are

$$
\begin{aligned}
& \ddot{U}=e^{2 U} V_{B H}, \\
& \ddot{z}^{a}+\Gamma_{b c}^{a} \dot{z}^{b} \dot{z}^{c}=e^{2 U} G^{a \bar{b}} \partial_{\bar{b}} V_{B H} .
\end{aligned}
$$

In addition, there is also a constraint on the system:

$$
\dot{U}^{2}+G_{a \bar{b}} \dot{z}^{a} \dot{\bar{z}}^{\bar{b}}-e^{2 U} V_{B H}=c^{2},
$$


where $c^{2}=2 S T=0$ for extremal black holes.

As described above, to generate solutions to these equations we take the horizon values of the moduli, (2.40), and replace the charges with appropriate harmonic functions:

$$
\begin{aligned}
e^{-2 U} & =2 \sqrt{H^{0} H_{1} H_{2} H_{3}} \\
z^{1} & =-i \sqrt{\frac{H_{2} H_{3}}{H^{0} H_{1}}} \\
z^{2} & =-i \sqrt{\frac{H_{1} H_{3}}{H^{0} H_{2}}} \\
z^{3} & =-i \sqrt{\frac{H_{1} H_{2}}{H^{0} H_{3}}} .
\end{aligned}
$$

Substitution followed by explicit calculation of the terms in the gravitational equation of motion gives:

$$
\begin{aligned}
\ddot{U} e^{-2 U}=V_{B H}= & \frac{1}{2}\left[\frac{q_{1}^{2}}{\left(H_{1}\right)^{3 / 2}} \sqrt{H^{0} H_{2} H_{3}}+\frac{q_{2}^{2}}{\left(H_{2}\right)^{3 / 2}} \sqrt{H^{0} H_{1} H_{3}}\right. \\
& \left.+\frac{q_{3}^{2}}{\left(H_{3}\right)^{3 / 2}} \sqrt{H^{0} H_{1} H_{2}}+\frac{\left(p^{0}\right)^{2}}{\left(H^{0}\right)^{3 / 2}} \sqrt{H_{1} H_{2} H_{3}}\right] .
\end{aligned}
$$

In a somewhat more involved calculation we can also verify that the ansatz solves the scalar equations of motion. Picking the 1 direction, we have:

$$
\begin{aligned}
\ddot{z}^{1}+\Gamma_{11}^{1}\left(\dot{z}^{1}\right)^{2} & =e^{2 U} G^{1 \overline{1}} \partial_{\overline{1}} V_{B H} \\
& =e^{2 U+K} G^{1 \overline{1}}\left(G^{2 \overline{2}}\left(\bar{D}_{\overline{1}} \bar{D}_{\overline{2}} \bar{W}\right) D_{2} W+G^{3 \overline{3}}\left(\bar{D}_{\overline{1}} \bar{D}_{\overline{3}} \bar{W}\right) D_{3} W+2\left(\bar{D}_{\overline{1}} \bar{W}\right) W\right)
\end{aligned}
$$

Once again substitution and tedium give the result we desire:

$$
\begin{aligned}
\ddot{z}^{1}+\Gamma_{11}^{1}\left(\dot{z}^{1}\right)^{2}= & e^{2 U} G^{1 \overline{1}} \partial_{\overline{1}} V_{B H} \\
= & \frac{i}{2}\left(\frac{H_{2} H_{3}}{H^{0} H_{1}}\right)^{-3 / 2}\left[q_{2}^{2}\left(H^{0} H_{1} H_{3}\right)^{2}+q_{3}^{2}\left(H^{0} H_{1} H_{2}\right)^{2}\right. \\
& \left.-q_{1}^{2}\left(H^{0} H_{2} H_{3}\right)^{2}-\left(p^{0}\right)^{2}\left(H_{1} H_{2} H_{3}\right)^{2}\right] .
\end{aligned}
$$

Finally, we check the constraint:

$$
\dot{U}^{2}+G_{a \bar{b}} \dot{z}^{a} \dot{\bar{z}}^{\bar{b}}=e^{2 U} V_{B H}=\frac{1}{4}\left[\frac{q_{1}^{2}}{H_{1}^{2}}+\frac{q_{2}^{2}}{H_{2}^{2}}+\frac{q_{3}^{2}}{H_{3}^{2}}+\frac{\left(p^{0}\right)^{2}}{\left(H^{0}\right)^{2}}\right] .
$$

\subsubsection{The Generic System: (D0,D2,D4,D6)}

To generalize the previous result, we need to establish that the prescription of the previous section, i.e. the substitution of harmonic functions for the electric and magnetic charges in the 
values of moduli at the horizon, solves the equations of motion when the moduli and metric are given by the expressions above:

$$
z^{\Lambda}(H(\mathbf{x}))=\frac{H^{\Lambda}-i \frac{\partial I_{1}(H)}{\partial H_{\Lambda}}}{H^{0}-i \frac{\partial I_{1}(H)}{\partial H_{0}}}, e^{-2 U}=\sqrt{|\mathcal{W}(\mathbf{H})|} .
$$

Given the hugely increased complexity of $I_{1}$ when all charges are present (not to mention $V_{B H}$ ) an explicit calculation would painful. Accordingly, we're going to do something else.

The symplectic invariance of special geometry ensures that the Lagrangian of our theory has an $S p(8, \mathbb{Z})$ symmetry, which reduces to $[S L(2, \mathbb{Z})]^{3}$ at the level of the equations of motion. This symmetry group can be used to generate solutions for generic charges by rotating the expressions for the moduli in the D2-D6 system (eq. (3.14)) obtained above. To do this we take the following element of $[S L(2, \mathbb{Z})]^{3}$ :

$$
\left(\begin{array}{ll}
a & b \\
c & d
\end{array}\right) \otimes\left(\begin{array}{ll}
1 & 0 \\
0 & 1
\end{array}\right) \otimes\left(\begin{array}{ll}
1 & 0 \\
0 & 1
\end{array}\right)
$$

in which $a d-b c=1$. Under this transformation, the charges and harmonic functions of the $D 2-D 6$ system transform in the following way

$$
\left(\begin{array}{c}
\tilde{p}^{0} \\
\tilde{p}^{1} \\
\tilde{p}^{2} \\
\tilde{p}^{3} \\
\tilde{q}_{0} \\
\tilde{q}_{1} \\
\tilde{q}_{2} \\
\tilde{q}_{3}
\end{array}\right)=\left(\begin{array}{c}
d p^{0} \\
b p^{0} \\
c q_{3} \\
c q_{2} \\
-b q_{1} \\
d q_{1} \\
a q_{2} \\
a q_{3}
\end{array}\right),\left(\begin{array}{c}
\tilde{H}^{0} \\
\tilde{H}^{1} \\
\tilde{H}^{2} \\
\tilde{H}^{3} \\
\tilde{H}_{0} \\
\tilde{H}_{1} \\
\tilde{H}_{2} \\
\tilde{H}_{3}
\end{array}\right)=\left(\begin{array}{c}
d H^{0} \\
b H^{0} \\
c H_{3} \\
c H_{2} \\
-b H_{1} \\
d H_{1} \\
a H_{2} \\
a H_{3}
\end{array}\right) .
$$

Further, we know that under transformation (3.20), the moduli coordinates transform as

$$
z^{1} \mapsto \tilde{z}^{1}=\frac{a z^{1}+b}{c z^{1}+d}, z^{2} \mapsto \tilde{z}^{2}=z^{2}, z^{3} \mapsto \tilde{z}^{3}=z^{3}
$$

The solution of equations of motion for the case of generic charges 4 can be swiftly obtained from (3.22).

To check whether (3.19) (with a set of generic charges) coincides with (3.22) it is sufficient to establish that (3.22) will solve the equations of motion. To do this we first we observe, as proved in [17], that the hyper-determinant $\mathcal{W}$ is invariant under (3.20). In our particular case this is simple to see from:

$$
\mathcal{W}(H) \mapsto \tilde{\mathcal{W}}(\tilde{H})=-4(a d-b c)^{2} H^{0} H_{1} H_{2} H_{3}=\mathcal{W}(H)
$$

\footnotetext{
4 We observe that the charges (and relevant harmonic function) are not completely independent and we have these two relations among them: $\tilde{p}^{3} \tilde{q}_{3}=\tilde{p}^{2} \tilde{q}_{2}$ and $\tilde{p}^{1} \tilde{q}_{1}=-\tilde{p}^{0} \tilde{q}_{0}$. If we also make nontrivial $S L(2, \mathbb{Z})$ transformations on the second and third moduli this dependence disappears. On the other hand, the above analysis shows that this procedure can be applied for successive transformations. Therefore, we do not lose the generality of the argument.
} 
Next, using (3.19), we obtain the modulus along the first direction of the moduli space for the generic case as

$$
\tilde{z}^{1}=\frac{\tilde{H}^{1} \sqrt{|\mathcal{W}|}+i\left(\tilde{H}^{1}\left(\tilde{H}^{2} \tilde{H}_{2}+\tilde{H}^{3} \tilde{H}_{3}-\tilde{H}^{0} \tilde{H}_{0}-\tilde{H}^{1} \tilde{H}_{1}\right)-2 \tilde{H}^{0} \tilde{H}_{2} \tilde{H}_{3}\right)}{\tilde{H}^{0} \sqrt{|\mathcal{W}|}+i\left(2 \tilde{H}^{1} \tilde{H}^{2} \tilde{H}^{3}-\tilde{H}^{0}\left(\tilde{H}^{\Lambda} \tilde{H}_{\Lambda}\right)\right)}
$$

This can be reexpressed in terms of the harmonic functions of the $D 2-D 6$ system:

$$
\tilde{z}^{1}=\frac{b \sqrt{H^{0} H_{1} H_{2} H_{3}}-i a H_{2} H_{3}}{d \sqrt{H^{0} H_{1} H_{2} H_{3}}-i c H_{2} H_{3}}=\frac{a\left(-i \sqrt{\frac{H_{2} H_{3}}{H^{0} H_{1}}}\right)+b}{c\left(-i \sqrt{\frac{H_{2} H_{3}}{H^{0} H_{1}}}\right)+d}=\frac{a z^{1}+b}{c z^{1}+d},
$$

where we have used the fact $a d-b c=1$ repeatedly. This is in complete agreement with (3.22). A similar check confirms that $\tilde{z}^{2}=z^{2}$ and $\tilde{z}^{3}=z^{3}$, as required. Hence, we can conclude that (3.19) gives the complete solutions to the equations of motion for stationary, spherically-symmetric non-BPS attractors. There is one small caveat, what we have actually done is produce a general solution from a restricted subset of $D 2-D 6$ attractors with $h_{0}=h^{1}=h^{2}=h^{3}=0$; while we believe that this simplification will not effect out result (it amounts to adding a constant electric/magnetic potential, something which clearly does not affect the vector equations of motion), we have been unable to check the equations of motion for the unconstrained D2-D6 case.

\section{Split Non-BPS Attractors}

So far we've managed to establish that many of the properties of STU non-BPS attractors are analogous to those of their BPS counterparts. In particular, along with the basic attractor behavior established in [4], we have also demonstrated explicitly (at least for the STU model) that complete expressions for the moduli can be formed from the attractor values, by replacing charges with harmonic functions. Given this success, it seems opportune to push a little further.

It has been shown in [17]-[19] and [27]-[29] that static, spherically-symmetric black holes are not the only BPS attractors one can find. Rather the attractor mechanism can be expanded to stationary spacetimes (dropping the requirement of spherical symmetry) with the metric:

$$
d s^{2}=-e^{2 U(\mathbf{x})}\left(d t+\omega_{i} d x^{i}\right)^{2}+e^{-2 U(\mathbf{x})} d x^{i} d x^{i}
$$

The most general situation described by this geometry is one consisting of multi-centered, charged black holes. Each black hole sits at a point $\vec{x}_{a}$ and carries a set of charges $\Gamma_{a}$. The vector fields are then fully described by a set of harmonic functions (a fact that doesn't rely on the BPS properties of the black holes, or the number of centers):

$$
H(\vec{x})=\left(H^{\Lambda}, H_{\Lambda}\right)=\mathbf{h}+\sum_{s=1}^{n} \frac{\Gamma_{s}}{\left|\vec{x}-\vec{x}_{s}\right|} .
$$


Bates and Denef have demonstrated (in [27]) that for the BPS case these harmonic functions can be used to construct exact solutions in an analogous fashion to the single center black holes. $\omega_{i}$ is defined through the harmonic functions:

$$
\omega_{i}=\langle\mathbf{H}, d \mathbf{H}\rangle .
$$

As before there is an integrability condition:

$$
\sum_{i=1}^{n} \frac{\left\langle\boldsymbol{\Gamma}_{\mathbf{j}}, \boldsymbol{\Gamma}_{i}\right\rangle}{\left|\vec{x}_{j}-\vec{x}_{i}\right|}+\left\langle\boldsymbol{\Gamma}_{j}, \mathbf{h}\right\rangle=0
$$

The above fixes the distance $\left|\vec{x}_{j}-\vec{x}_{i}\right|$ unless the charges are mutually local, with $\left\langle\boldsymbol{\Gamma}_{\mathbf{i}}, \boldsymbol{\Gamma}_{\mathbf{j}}\right\rangle=0$ - this is the case when $\omega_{i}=0$. We also have a similar constraint on $\mathbf{h}$ as for the single center attractor: $\langle\mathbf{h}, \boldsymbol{\Gamma}\rangle=0$, where $\boldsymbol{\Gamma}=\sum_{i} \boldsymbol{\Gamma}_{i}$. In this setup the BPS solutions are given by:

$$
\begin{aligned}
z^{\Sigma}(H(\vec{x})) & =\frac{H^{\Sigma}+i \frac{\partial I_{1}(H)}{\partial H_{\Sigma}}}{H^{0}+i \frac{\partial I_{1}(H)}{\partial H_{0}}}, \\
e^{-2 U} & =I_{1}(\mathbf{H}) .
\end{aligned}
$$

Where we define the symplectic invariant $I_{1}$ as before:

$$
I_{1}(H)=|Z(H)|^{2}+|D Z(H)|^{2}=\sqrt{|\mathcal{W}(H)|} \geq 0,
$$

For the non-BPS STU model we thus conjecture that, again analogous to the single center black hole, solutions are given by:

$$
\begin{aligned}
z^{\Sigma}(H(\vec{x})) & =\frac{H^{\Sigma}-i \frac{\partial I_{1}(H)}{\partial H_{\Sigma}}}{H^{0}-i \frac{\partial I_{1}(H)}{\partial H_{0}}}, \\
e^{-2 U} & =I_{1}(\mathbf{H})
\end{aligned}
$$

Of course, here $\mathcal{W}(\mathbf{H})<0$. Our principle motivation here, once again, is the underlying duality symmetry and the constraining restrictions it must place on the form of any solution. In theory, we can check this solution in an entirely similar fashion to the spherically-symmetric cases discussed above. However, the process is somewhat more involved, and as yet it has not proved possible to carry out the required calculations.

There are, though, good reasons to believe that multi-centered, non-BPS, extremal black hole attractors exist; and that the behavior of the moduli fields is described by (4.7). As discussed in [4] and [16] the attractiveness of black holes is a result of their extremality. Specifically, extremal black holes have a near horizon geometry described by a Bertotti-Robinson $\left(A d S_{2} \times S^{2}\right)$ product space with an infinite throat. It is this infinite throat that leads to attractor behavior and it should thus be clear that if extremal multi-centered stationary solutions can be found, then each black hole will have the same near-horizon behavior as it would do in isolation. Accordingly, we should expect each horizon to be an attractor. Furthermore, it is apparent that solutions for $z^{a}$ of the form (4.7) will reduce to the single-center ones in the 
near-horizon limit - suggesting that they may well be the appropriate global expressions for the moduli. The missing piece in this argument, and the reason one would want to check equations of motion explicitly, is that we cannot be certain that stationary, multi-centered solutions exist for extremal, non-BPS black holes.

Before moving on to a discussion of our results, there is one last observation that we should make. Denef has observed in [28] that for a multi-center (charges $\Gamma_{a}$ ) BPS black hole the behavior far from all centers should be that of a spherically symmetric solution with charge $\Gamma=\sum_{a} \Gamma_{a}$, leading to a split attractor flow. The same should hold true for non-BPS case, though here we could have an interesting additional effect. Whilst BPS extremal black holes are always stable, non-BPS ones are not - so although systems with $\Gamma_{1}=\left(q_{0}, 0,0, p^{a}\right)$ and those with $\Gamma_{2}=\left(0, q_{a}, p^{0}, 0\right)$ give stable attractors, systems with $\Gamma=\Gamma_{1}+\Gamma_{2}=\left(q_{0}, q_{a}, p^{0}, p^{a}\right)$ do not. Thus we might expect that apparently unstable "attractors" correspond to stable multi-centered configurations when examined at appropriately short distances from the horizon 5 .

\section{Discussion}

In the preceding sections we have discussed some of issues arising in non-BPS STU attractors. In particular we have found that the system consisting of $p^{0}$ and $q_{a}$ charges can give rise to a stable non-BPS attractor - a result that was obtained using both the attractor equation (in two different forms) and through the minimization of an effective black hole potential. Further we have demonstrated that one can construct exact expressions for the moduli, the metric and the vector fields using harmonic functions through a prescription analogous to that used for BPS black holes.

Additionally, we have argued for the existence of stationary non-BPS attractors, with an associated split attractor flow. This allows us to make the following conjecture regarding unstable STU non-BPS "attractors":

Conjecture. The apparent unstable nature of non-BPS STU "attractors" can be explained through the existence of a stable multi-center solution that is only resolved at sufficiently short distances. It is only far away from both centers that the gradient flow of the system appears repulsive.

We hope to present a proof of this conjecture in future work.

Finally, we note that while everything we have discussed only applies to STU black holes, we see no reason in principle why the behavior we have found and the arguments we have made for both exact solutions and multi-center attractors will not apply to more general extremal non-BPS black holes.

\footnotetext{
${ }^{5}$ There is some additional subtlety here - since the gradient flow will have to switch direction when the flow splits we should expect the line of marginal stability ([29], [28]), where the split takes place, to have somewhat different properties to that in the BPS case.
} 


\section{Acknowledgments}

This work is supported by NSF grant 0244728. N.S. and M.S. are also supported by the U.S. Department of Energy under contract number DE-AC02-76SF00515.

\section{A Further Details of the $D 2-D 6$ system}

In this appendix we discuss various details of the $D 2-D 6$ system - developing the solution established above by confirming it through some alternate methods and analyzing its stability.

\section{A.1 Minimizing the Effective Potential}

Although we calculated the values of moduli at the black hole horizon by using the attractor equation we can, instead, directly extremize the effective potential; i.e. solve $\partial_{a} V_{B H}=0$ :

$$
\partial_{a} V_{B H}=e^{K}\left(G^{b \bar{c}}\left(D_{a} D_{b} W\right) \bar{D}_{\bar{c}} \bar{W}+2\left(D_{a} W\right) \bar{W}\right) .
$$

Since the superpotential only includes odd powers of the coordinates for the system of $D 2-D 6$ brane, we assume that the ansatz of the minimization of the black hole potential equation is purely imaginary $\bar{z}^{a}=-z^{a}$ (see [4]). Now, we want to compute the ingredients of (A.1). The first covariant derivative of the superpotential is:

$$
D_{a} W=\left(\partial_{a}+\partial_{a} K\right) W=-\frac{W\left(z^{1}, \cdots, \bar{z}^{a}, \cdots, z^{3}\right)}{z^{a}-\bar{z}^{a}} .
$$

Note that in the above expression, $\bar{z}^{a}$ is substituted in the argument of the superpotential for $z^{a}$. With the ansatz $\bar{z}^{a}=-z^{a}$, (A.2) then gives:

$$
\begin{aligned}
& D_{1} W=-\frac{W\left(\bar{z}^{1}, z^{2}, z^{3}\right)}{z^{1}-\bar{z}^{1}}=\frac{W-2\left(q_{2} z^{2}+q_{3} z^{3}\right)}{2 z^{1}}, \\
& D_{2} W=-\frac{W\left(z^{1}, \bar{z}^{2}, z^{3}\right)}{z^{2}-\bar{z}^{2}}=\frac{W-2\left(q_{1} z^{1}+q_{3} z^{3}\right)}{2 z^{2}}, \\
& D_{3} W=-\frac{W\left(z^{1}, z^{2}, \bar{z}^{3}\right)}{z^{3}-\bar{z}^{3}}=\frac{W-2\left(q_{1} z^{1}+q_{2} z^{2}\right)}{2 z^{3}} .
\end{aligned}
$$

When we drop the arguments of the superpotential they should be understood to be $z^{1}, z^{2}$ and $z^{3}$. Before we consider the non-SUSY solutions, we find the supersymmetric ones, $D_{a} W=0$ :

$$
z^{1}=i \sqrt{-\frac{q_{2} q_{3}}{p^{0} q_{1}}}, z^{2}=i \sqrt{-\frac{q_{1} q_{3}}{p^{0} q_{2}}}, z^{3}=i \sqrt{-\frac{q_{1} q_{2}}{p^{0} q_{3}}},
$$

and also

$$
z^{1}=-i \sqrt{-\frac{q_{2} q_{3}}{p^{0} q_{1}}}, z^{2}=-i \sqrt{-\frac{q_{1} q_{3}}{p^{0} q_{2}}}, z^{3}=-i \sqrt{-\frac{q_{1} q_{2}}{p^{0} q_{3}}} .
$$


As briefly discussed above, we should examine these solutions to see whether they respect the positivity of the Kähler potential - a necessary condition to ensure positive kinetic terms in the action. A simple check shows that the first SUSY solution (A.6) violates the positivity of the Kähler potential and therefore, it is ruled out. Thus only the latter (A.7) is acceptable. We should also notice that these solutions will only exist when $p^{0} q_{1} q_{2} q_{3}<0$.

The next object to calculate is the second covariant derivative of $W$ :

$$
D_{a} D_{b} W=\left(\partial_{a}+\partial_{a} K\right) D_{b} W-\Gamma_{a b}^{c} D_{c} W
$$

For our particular STU model:

$$
D_{a} D_{b} W=\left(1-\delta_{a b}\right) \frac{W\left(z^{1}, \cdots, \bar{z}^{a}, \bar{z}^{b}, \cdots\right)}{\left(z^{a}-\bar{z}^{a}\right)\left(z^{b}-\bar{z}^{b}\right)} .
$$

Notice that $\bar{z}^{a}$ and $\bar{z}^{b}$ substitute $z^{a}$ and $z^{b}$ in the argument of $W$. For the $z^{a}=-\bar{z}^{a}$ ansatz of our $D 2-D 6$ brane system, the above reduces to:

$$
\left.D_{a} D_{b} W=\left(1-\delta_{a b}\right) \frac{W-2\left(q_{a} z^{a}+q_{b} z^{b}\right)}{4 z^{a} z^{b}} \text { (no sum on } a \text { and } b\right) .
$$

Now, we are ready to form the equation ofan explicit expression for the minimization of the potential $\partial_{a} V_{B H}=0$. Using (2.8), (A.3), and (A.10), we find the following set of equations:

$$
\begin{aligned}
& \left(W-2\left(q_{1} z^{1}+q_{2} z^{2}\right)\right)\left(W-2\left(q_{1} z^{1}+q_{3} z^{3}\right)\right)+W\left(W-2\left(q_{2} z^{2}+q_{3} z^{3}\right)\right)=0, \\
& \left(W-2\left(q_{1} z^{1}+q_{2} z^{2}\right)\right)\left(W-2\left(q_{2} z^{2}+q_{3} z^{3}\right)\right)+W\left(W-2\left(q_{1} z^{1}+q_{3} z^{3}\right)\right)=0 \\
& \left(W-2\left(q_{1} z^{1}+q_{3} z^{3}\right)\right)\left(W-2\left(q_{2} z^{2}+q_{3} z^{3}\right)\right)+W\left(W-2\left(q_{1} z^{1}+q_{2} z^{2}\right)\right)=0 .
\end{aligned}
$$

It is clear that (A.7) satisfy these equations. Now, we want to find the non-SUSY solutions $\left(D_{a} W \neq 0\right)$. If we divide the above expressions by one another, we obtain:

$$
\left(W-2\left(q_{1} z^{1}+q_{2} z^{2}\right)\right)^{2}=\left(W-2\left(q_{2} z^{2}+q_{3} z^{3}\right)\right)^{2}=\left(W-2\left(q_{1} z^{1}+q_{3} z^{3}\right)\right)^{2} .
$$

The complete solutions to these relations are:

$$
z^{1}= \pm i \sqrt{\frac{q_{2} q_{3}}{p^{0} q_{1}}}, z^{2}= \pm i \sqrt{\frac{q_{1} q_{3}}{p^{0} q_{2}}}, z^{3}= \pm i \sqrt{\frac{q_{1} q_{2}}{p^{0} q_{3}}}
$$

If we consider all these possibilities for (A.14), then we find eight sets, which can be categorized into two groups:

$$
\begin{aligned}
& A:\{(+,+,+),(+,-,-),(-,+,-),(-,-,+)\}, \\
& B:\{(-,-,-),(-,+,+),(+,-,+),(+,+,-)\} .
\end{aligned}
$$

In above, each parenthesis shows the sign of $\left(z^{1}, z^{2}, z^{3}\right)$ respectively. It turns out that the elements of group $A$ violate the positivity of $e^{K}$ and therefore this group is unacceptable. 
However, no such problem exists for group $B$, and therefore its elements provide acceptable non-SUSY solutions. Furthermore, the last three elements of group $B$ are physically equivalent solutions in which the sign of two fields are the same, but opposite to the third. It's worth noting that that the attractor equation gave us only the $(-,-,-)$ solution; however, this point has little effect on the rest of out argument.

Finally, we note that for all non-SUSY solutions, we must have $p^{0} q_{1} q_{2} q_{3}>0$. This in turn means that we cannot simultaneously have both SUSY and non-SUSY solutions.

\section{A.2 An Alternate Attractor Equation}

In this section, we find the values of the moduli at the horizon of the black hole by solving the attractor equation in the form given in [16]:

$$
\Gamma=2 \operatorname{Im}\left[Z \bar{\Pi}-\frac{\left(\bar{D}_{\bar{a}} \bar{D}_{\bar{b}} \bar{Z}\right) G^{\bar{a} c} G^{\bar{b} d} D_{c} Z}{2 Z} D_{d} \Pi\right]
$$

As before $\Gamma$ is the set of magnetic and electric charges $\Gamma=\left(p^{\Lambda}, q_{\Lambda}\right)$ and $\Pi$ is the covariantly holomorphic period vector:

$$
\Pi=e^{K / 2}\left(\begin{array}{c}
1 \\
z^{a} \\
F_{0} \\
F_{a}
\end{array}\right)
$$

We have already calculated most of these ingredients, with our only remaining task to calculate the covariant derivative of $\Pi$ :

$$
D_{d} \Pi=e^{K / 2}\left(\begin{array}{c}
\partial_{d} K \\
\delta_{d}^{a}+\left(\partial_{d} K\right) z^{a} \\
\partial_{d} F_{0}+\left(\partial_{d} K\right) F_{0} \\
\partial_{d} F_{a}+\left(\partial_{d} K\right) F_{a}
\end{array}\right)
$$

It is straightforward to calculate the two terms of the attractor equation for our ansatz $\bar{z}^{a}=-z^{a}$. These are:

$$
2 \operatorname{Im}(Z \bar{\Pi})=\frac{W}{4 z^{1} z^{2} z^{3}}\left(\begin{array}{c}
1 \\
0 \\
0 \\
0 \\
0 \\
z^{2} z^{3} \\
z^{1} z^{3} \\
z^{1} z^{2}
\end{array}\right), 2 \operatorname{Im}\left[\frac{\left(\bar{D}_{\bar{a}} \bar{D}_{\bar{b}} \bar{Z}\right) G^{\bar{a} c} G^{\bar{b} d} D_{c} Z}{2 Z} D_{d} \Pi\right]=-\frac{1}{4 z^{1} z^{2} z^{3}} \frac{1}{W}\left(\begin{array}{c}
Y_{0} \\
0 \\
0 \\
0 \\
0 \\
Y_{1} z^{2} z^{3} \\
Y_{2} z^{1} z^{3} \\
Y_{3} z^{1} z^{2}
\end{array}\right) .
$$


We have defined $Y_{0}, Y_{1}, Y_{2}$, and $Y_{3}$ to be:

$$
\begin{aligned}
& Y_{0} \equiv W\left(\bar{z}^{1}, z^{2}, z^{3}\right) W\left(z^{1}, \bar{z}^{2}, z^{3}\right)+W\left(\bar{z}^{1}, z^{2}, z^{3}\right) W\left(z^{1}, z^{2}, \bar{z}^{3}\right)+W\left(z^{1}, \bar{z}^{2}, z^{3}\right) W\left(z^{1}, z^{2}, \bar{z}^{3}\right) \\
& Y_{1} \equiv W\left(\bar{z}^{1}, z^{2}, z^{3}\right) W\left(z^{1}, \bar{z}^{2}, z^{3}\right)+W\left(\bar{z}^{1}, z^{2}, z^{3}\right) W\left(z^{1}, z^{2}, \bar{z}^{3}\right)-W\left(z^{1}, \bar{z}^{2}, z^{3}\right) W\left(z^{1}, z^{2}, \bar{z}^{3}\right) \\
& Y_{2} \equiv W\left(\bar{z}^{1}, z^{2}, z^{3}\right) W\left(z^{1}, \bar{z}^{2}, z^{3}\right)+W\left(z^{1}, \bar{z}^{2}, z^{3}\right) W\left(z^{1}, z^{2}, \bar{z}^{3}\right)-W\left(\bar{z}^{1}, z^{2}, z^{3}\right) W\left(z^{1}, z^{2}, \bar{z}^{3}\right) \\
& Y_{3} \equiv W\left(\bar{z}^{1}, z^{2}, z^{3}\right) W\left(z^{1}, z^{2}, \bar{z}^{3}\right)+W\left(z^{1}, \bar{z}^{2}, z^{3}\right) W\left(z^{1}, z^{2}, \bar{z}^{3}\right)-W\left(\bar{z}^{1}, z^{2}, z^{3}\right) W\left(z^{1}, \bar{z}^{2}, z^{3}\right) .
\end{aligned}
$$

Forming the attractor equation (A.18), we find the following:

$$
\begin{aligned}
& W^{2}-Y_{0}=4 p^{0} W z^{1} z^{2} z^{3} \\
& W^{2}+Y_{a}=4 q_{a} W z^{a}(\text { no sum on } a)
\end{aligned}
$$

As is clear, we have four equations, but only three of them are independent. This can be easily seen if we add the four to one another and use $Y_{1}+Y_{2}+Y_{3}=Y_{0}$. To solve these equations we first note that $Y_{1}-4 q_{1} W z^{1}=Y_{2}-4 q_{2} W z^{2}=Y_{3}-4 q_{3} W z^{3}$ and observe this leads to:

$$
q_{1} z^{1}= \pm q_{2} z^{2}= \pm q_{3} z^{3}
$$

If we take $q_{1} z^{1}=q_{2} z^{2}=q_{3} z^{3}$ we find:

$$
\left(z^{1}-\frac{p^{0} q_{1}}{q_{2} q_{3}}\left(z^{1}\right)^{3}\right)\left(z^{1}+\frac{p^{0} q_{1}}{q_{2} q_{3}}\left(z^{1}\right)^{3}\right)=0 .
$$

We immediately realize that the first parenthesis produces the supersymmetric solution:

$$
z^{1}= \pm i \sqrt{-\frac{q_{2} q_{3}}{p^{0} q_{1}}}
$$

The second parenthesis of (A.26) then gives us the non-supersymmetric attractor:

$$
z^{1}= \pm i \sqrt{\frac{q_{2} q_{3}}{p^{0} q_{1}}} .
$$

As we see, different choices of (A.25) produce all the different SUSY and non-SUSY solutions that we found in the previous section. Recalling that the positivity of $e^{K}$ requires we choose a specific set of signs for the above solutions leads to (A.7) for the supersymmetric solution and elements of group $B$ (see A.16) for the non-supersymmetric ones.

\section{A.3 Stability}

In the previous section, we found both SUSY and non-SUSY attractors. The former are always stable, since the second derivative of the black hole potential is proportional to the Kähler metric. However, for non-SUSY solutions, we need to check stability explicitly. In order to do this, we need to find the full mass matrix. 
First, note that at the extremum of the potential we have $\partial_{a} \partial_{b} V_{B H}=D_{a} D_{b} V_{B H}$; therefore, we can use the covariant rather than ordinary derivative. Then the holomorphic-holomorphic and holomorphic-antiholomorphic parts of the mass matrix are given by

$$
\begin{aligned}
D_{a} D_{b} V_{B H}= & e^{K}\left(G^{c \bar{d}}\left(D_{a} D_{b} D_{c} W\right) \bar{D}_{\bar{d}} \bar{W}+3\left(D_{a} D_{b} W\right) \bar{W}\right) \\
\bar{D}_{\bar{a}} D_{b} V_{B H}= & e^{K}\left(-\mathcal{R}_{b \bar{a} c}^{d} G^{c \bar{e}}\left(D_{d} W\right) \bar{D}_{\bar{e}} \bar{W}+G^{c \bar{d}}\left(D_{b} D_{c} W\right)\left(\bar{D}_{\bar{a}} \bar{D}_{\bar{d}} \bar{W}\right)\right. \\
& \left.+G_{\bar{a} b} G^{c \bar{d}}\left(D_{c} W\right) \bar{D}_{\bar{d}} \bar{W}+3\left(\bar{D}_{\bar{a}} \bar{W}\right) D_{b} W+2 G_{\bar{a} b} W \bar{W}\right),
\end{aligned}
$$

where $\mathcal{R}_{b \bar{a} c}^{d}$ is the Riemann curvature tensor of the moduli space. We begin with the holomorphicholomorphic piece. This requires us to compute the third covariant derivative of the superpotential:

$$
\left.D_{a} D_{b} D_{c} W=-\left|\epsilon_{a b c}\right| \frac{W\left(\bar{z}^{1}, \bar{z}^{2}, \bar{z}^{3}\right)}{\left(z^{a}-\bar{z}^{a}\right)\left(z^{b}-\bar{z}^{b}\right)\left(z^{c}-\bar{z}^{c}\right)}, \quad \text { (no sum on } a, b, \text { and } c\right) .
$$

Using this result and defining $\left.B_{a b} \equiv D_{a} D_{b} V_{B H}\right|_{\text {horizon }}$ we have:

$$
B=\frac{1}{2} p^{0} \sqrt{p^{0} q_{1} q_{2} q_{3}}\left(\begin{array}{ccc}
0 & \frac{1}{q_{3}} & \frac{1}{q_{2}} \\
\frac{1}{q_{3}} & 0 & \frac{1}{q_{1}} \\
\frac{1}{q_{2}} & \frac{1}{q_{1}} & 0
\end{array}\right) .
$$

To find the antiholomorphic-holomorphic piece of the mass matrix, we need the Riemann curvature of the moduli space:

$$
\mathcal{R}_{a \bar{a} a}^{a}=-\frac{2}{\left(z^{a}-\bar{z}^{a}\right)^{2}}, \quad(\text { no sum on } a) .
$$

All other components of the Riemann curvature tensor vanish. Taking this result we can crawl through a long computation to obtain $A_{a b}=\left.\bar{D}_{\bar{a}} D_{b} V_{B H}\right|_{\text {horizon }}$ as:

$$
A=\frac{1}{2} p^{0} \sqrt{p^{0} q_{1} q_{2} q_{3}}\left(\begin{array}{ccc}
\frac{2 q_{1}}{q_{2} q_{3}} & \frac{1}{q_{3}} & \frac{1}{q_{2}} \\
\frac{1}{q_{3}} & \frac{q_{2}}{q_{1} q_{3}} & \frac{1}{q_{1}} \\
\frac{1}{q_{2}} & \frac{1}{q_{1}} & \frac{2 q_{3}}{q_{1} q_{2}}
\end{array}\right) .
$$

Hence, the full mass matrix of the black hole potential is given by:

$$
M=\left(\begin{array}{cc}
A & \bar{B} \\
B & \bar{A}
\end{array}\right)=\frac{1}{2} p^{0} \sqrt{p^{0} q_{1} q_{2} q_{3}}\left(\begin{array}{cccccc}
\frac{2 q_{1}}{q_{2} q_{3}} & \frac{1}{q_{3}} & \frac{1}{q_{2}} & 0 & \frac{1}{q_{3}} & \frac{1}{q_{2}} \\
\frac{1}{q_{3}} & \frac{2 q_{2}}{q_{1} q_{3}} & \frac{1}{q_{1}} & \frac{1}{q_{3}} & 0 & \frac{1}{q_{1}} \\
\frac{1}{q_{2}} & \frac{1}{q_{1}} & \frac{2 q_{3}}{q_{2} q_{2}} & \frac{1}{q_{2}} & \frac{1}{q_{1}} & 0 \\
0 & \frac{1}{q_{3}} & \frac{1}{q_{2}} & \frac{2 q_{1}}{q_{2} q_{3}} & \frac{1}{q_{3}} & \frac{1}{q_{2}} \\
\frac{1}{q_{3}} & 0 & \frac{1}{q_{1}} & \frac{1}{q_{3}} & \frac{2 q_{2}}{q_{1} q_{3}} & \frac{1}{q_{1}} \\
\frac{1}{q_{2}} & \frac{1}{q_{1}} & 0 & \frac{1}{q_{2}} & \frac{1}{q_{1}} & \frac{2 q_{3}}{q_{1} q_{2}}
\end{array}\right) .
$$

Note that this will give rise to a term in the effective potential which takes the following form:

$$
z^{\dagger} M z \quad \text { where, } \quad z=\left(z^{1}, z^{2}, z^{3}, \bar{z}^{1}, \bar{z}^{2}, \bar{z}^{3}\right) .
$$


To explore the stability of the non-SUSY solutions we need to diagonalize the mass matrix. This is straightforward and the eigenvalues are given by:

$$
\left\{0,0, \sqrt{p^{0} q_{1} q_{2} q_{3}} \frac{p^{0} q_{1}}{q_{2} q_{3}}, \sqrt{p^{0} q_{1} q_{2} q_{3}} \frac{p^{0} q_{2}}{q_{1} q_{3}}, \sqrt{p^{0} q_{1} q_{2} q_{3}} \frac{p^{0} q_{3}}{q_{1} q_{2}}, \sqrt{p^{0} q_{1} q_{2} q_{3}}\left(\frac{p^{0} q_{1}}{q_{2} q_{3}}+\frac{p^{0} q_{2}}{q_{1} q_{3}}+\frac{p^{0} q_{3}}{q_{1} q_{2}}\right)\right\}(
$$

There are two flat directions, with the rest of the eigenvalues positive. For the former we find that the associated eigenvectors are:

$$
\left(-\frac{q_{3}}{q_{1}}, 0,1,-\frac{q_{3}}{q_{1}}, 0,1\right) \quad \text { and } \quad\left(-\frac{q_{2}}{q_{1}}, 1,0,-\frac{q_{2}}{q_{1}}, 1,0\right) .
$$

Both of these (as is clear from (A.36) $)$ are in a real direction - moving along potential in the direction of the eigenvectors sends $z^{a} \rightarrow z^{a}+\delta x^{a}$, where $\delta x^{a}$ is real.

In order to explore the stability of our extrema with respect to the flat directions, we need to examine higher order derivatives. Recall that we initially proceeded with covariant derivatives instead of ordinary derivatives (since $D D V_{B H}=d^{2} V_{B H}$ at extremal points of $V_{B H}$ ). But this is no longer true at higher orders, so one needs to revert to the flat derivative. Unfortunately, this becomes rather unpleasant, as we forced to break the covariant form, hence we choose to proceed in a calculationally more tractable fashion: We expand the black hole potential around the non-supersymmetric solutions and then concentrate on the behavior of cubic and quartic terms of the expansion. If the coefficients of the cubic terms are non-vanishing, then we can conclude that the non-supersymmetric solution is unstable. However, if the coefficients vanish, then we need to consider the quartic terms. If these terms are positive for flat directions, independent of the values of the parameter of the expansion, then the non-supersymmetric solution is indeed stable.

First, using (2.8) and (A.3), we notice that we can rewrite the black hole potential in the following way

$$
V_{B H}=e^{K}\left(\left|W\left(\bar{z}^{1}, z^{2}, z^{3}\right)\right|^{2}+\left|W\left(z^{1}, \bar{z}^{2}, z^{3}\right)\right|^{2}+\left|W\left(z^{1}, z^{2}, \bar{z}^{3}\right)\right|^{2}+\left|W\left(z^{1}, z^{2}, z^{3}\right)\right|^{2}\right) .
$$

Now, we want to expand the above potential around the non-supersymmetric solution (A.15) as

$$
z^{1}=-i \sqrt{\frac{q_{2} q_{3}}{p^{0} q_{1}}}+\epsilon^{1}, \quad z^{2}=-i \sqrt{\frac{q_{1} q_{3}}{p^{0} q_{2}}}+\epsilon^{2}, \quad z^{3}=-i \sqrt{\frac{q_{1} q_{2}}{p^{0} q_{3}}}+\epsilon^{3},
$$

where $\epsilon^{a}$ is a real parameter of the expansion. Why only real? Well we are, of course, only concerned with the quartic expansion in the flat directions, and we have already established that, for our first non-supersymmetric solution, these are real. Accordingly $V_{B H}$ has the following expansion

$$
V_{B H}\left(z^{a}\right)=V_{B H}\left(z_{0}^{a}\right)+\mathcal{O}\left(\left(\epsilon^{a}\right)^{2}\right)+\mathcal{O}\left(\left(\epsilon^{a}\right)^{3}\right)+\mathcal{O}\left(\left(\epsilon^{a}\right)^{4}\right)+\cdots
$$

where $z_{0}^{a}$ is the value of moduli for non-supersymmetric solution. Note that the flat directions in (A.38) correspond to the plane $q_{1} \epsilon^{1}+q_{2} \epsilon^{2}+q_{3} \epsilon^{3}=0$ in notation of (A.40). 
The information in $\mathcal{O}\left(\left(\epsilon^{a}\right)^{2}\right)$ is encoded in the mass matrix which has already been calculated and we need to know about higher order terms along flat directions. Explicit computation shows that the cubic terms vanish $\mathcal{O}\left(\left(\epsilon^{a}\right)^{3}\right)=0$. Thus we must examine the quartic terms and we find:

$$
\begin{aligned}
\mathcal{O}\left(\left(\epsilon^{a}\right)^{4}\right)= & 4 e^{K\left(z_{0}^{a}\right)} p^{0}\left(\frac{q_{1} q_{2}}{q_{3}}\left(\epsilon^{1} \epsilon^{2}\right)^{2}+\frac{q_{2} q_{3}}{q_{1}}\left(\epsilon^{2} \epsilon^{3}\right)^{2}+\frac{q_{1} q_{3}}{q_{2}}\left(\epsilon^{1} \epsilon^{3}\right)^{2}\right. \\
& \left.+q_{1}\left(\epsilon^{1}\right)^{2} \epsilon^{2} \epsilon^{3}+q_{2} \epsilon^{1}\left(\epsilon^{2}\right)^{2} \epsilon^{3}+q_{3} \epsilon^{1} \epsilon^{2}\left(\epsilon^{3}\right)^{2}\right) \\
= & 2 e^{K\left(z_{0}^{a}\right)} \frac{\left(p^{0}\right)^{2}}{p^{0} q_{1} q_{2} q_{3}}\left[a_{1}^{2}+a_{2}^{2}+a_{3}^{2}+\left(a_{1}+a_{2}+a_{3}\right)^{2}\right] .
\end{aligned}
$$

Here $a_{1}=q_{2} \epsilon^{2} q_{3} \epsilon^{3}, a_{2}=q_{1} \epsilon^{1} q_{3} \epsilon^{3}$ and $a_{3}=q_{1} \epsilon^{1} q_{2} \epsilon^{2}$. As we see, no matter how the parameters of expansion change, $\mathcal{O}\left(\left(\epsilon^{a}\right)^{4}\right)$ is manifestly positive.

This implies that the non-SUSY solution (A.15) is indeed stable. Finally, we mention that it can be (and has been) checked that the other non-SUSY solution $(-,+,+)$ also gives a positive mass matrix.

\section{References}

[1] S. Ferrara, R. Kallosh and A. Strominger, "N=2 extremal black holes," Phys. Rev. D 52, 5412 (1995) arXiv:hep-th/9508072.

[2] A. Strominger, "Macroscopic Entropy of $N=2$ Extremal Black Holes," Phys. Lett. B 383, 39 (1996) arXiv:hep-th/9602111.

[3] S. Ferrara and R. Kallosh, "Supersymmetry and Attractors," Phys. Rev. D 54, 1514 (1996) arXiv:hep-th/9602136]; S. Ferrara and R. Kallosh, "Universality of Supersymmetric Attractors," Phys. Rev. D 54, 1525 (1996) arXiv:hep-th/9603090].

[4] K. Goldstein, N. Iizuka, R. P. Jena and S. P. Trivedi, "Non-supersymmetric attractors," arXiv:hep-th/0507096;

[5] P. K. Tripathy and S. P. Trivedi, "Non-Supersymmetric Attractors in String Theory," arXiv:hep-th/0511117.

[6] R. Kallosh, "New attractors," JHEP 0512, 022 (2005) arXiv:hep-th/0510024.

[7] A. Giryavets, "New attractors and area codes," JHEP 0603, 020 (2006) arXiv:hep-th/0511215.

[8] S. Ferrara and R. Kallosh, "On N = 8 attractors," Phys. Rev. D 73, 125005 (2006) arXiv:hep-th/0603247.

${ }^{6}$ This result is no longer true in the presence of D4 branes. In fact, the details of the calculations show that if all existing terms in the superpotential either have even or odd powers of the moduli coordinates, then the cubic term $\mathcal{O}\left(\left(\epsilon^{a}\right)^{3}\right)$ (which is the leading term in perturbation) vanishes. But in general case when the superpotential has a mixture of even and odd powers, then the cubic term does not vanish and therefore, the extremum is an inflection point rather than a minimum. 
[9] S. Ferrara, E. G. Gimon and R. Kallosh, "Magic Supergravities, N=8 and Black Hole Composites," arXiv:hep-th/0606211.

[10] S. Bellucci, S. Ferrara and A. Marrani, "On some properties of the attractor equations," Phys. Lett. B 635, 172 (2006) [arXiv:hep-th/0602161].

[11] S. Bellucci, S. Ferrara, M. Gunaydin and A. Marrani, "Charge Orbits of Symmetric Special Geometries and Attractors," arXiv:hep-th/0606209.

[12] R. Kallosh, "From BPS to non-BPS black holes canonically," arXiv:hep-th/0603003.

[13] R. Kallosh and A. Linde, "Strings, black holes, and quantum information," Phys. Rev. D 73, 104033 (2006) arXiv:hep-th/0602061.

[14] B. Sahoo and A. Sen, "Higher derivative corrections to non-supersymmetric extremal black holes in arXiv:hep-th/0603149; A. Sen, "Black hole entropy function and the attractor mechanism in higher derivative gravity," JHEP 0509, 038 (2005) arXiv:hep-th/0506177]; M. Alishahiha and H. Ebrahim, "New attractor, entropy function and black hole partition function," arXiv:hep-th/0605279; S. Parvizi and A. Tavanfar, "Partition function of non-supersymmetric black holes in the supergravity arXiv:hep-th/0602292.

D. Astefanesei, K. Goldstein, R. P. Jena, A. Sen and S. P. Trivedi, "Rotating Attractors," arXiv:hep-th/0606244.

[15] D. Green, E. Silverstein and D. Starr, "Attractor explosions and catalyzed vacuum decay," arXiv:hep-th/0605047.

[16] R. Kallosh, N. Sivanandam and M. Soroush, "The non-BPS black hole attractor equation," JHEP 0603, 060 (2006) arXiv:hep-th/0602005].

[17] K. Behrndt, D. Lust and W. A. Sabra, "Stationary solutions of N = 2 supergravity," Nucl. Phys. B 510, 264 (1998) arXiv:hep-th/9705169.

[18] W. A. Sabra, "Black holes in N=2 supergravity theories and harmonic functions," Nucl. Phys. B 510, 247 (1998) arXiv:hep-th/9704147.

[19] W. A. Sabra, "General static N = 2 black holes," Mod. Phys. Lett. A 12, 2585 (1997) arXiv:hep-th/9703101.

[20] P. Breitenlohner, D. Maison and G. Gibbons "4-Dimensional Black Holes from KaluzaKlein Theories," Commun. Math. Phys. 120, 295 (1988)

[21] K. Behrndt, R. Kallosh, J. Rahmfeld, M. Shmakova and W. K. Wong, "STU black holes and string triality," Phys. Rev. D 54, 6293 (1996) arXiv:hep-th/9608059.

[22] L. Andrianopoli, R. D'Auria, S. Ferrara, P. Fre and M. Trigiante, "E(7)(7) duality, BPS black-hole evolution and fixed scalars," Nucl. Phys. B 509, 463 (1998) arXiv:hep-th/9707087.

[23] S. Ferrara, G. W. Gibbons and R. Kallosh, "Black holes and critical points in moduli space," Nucl. Phys. B 500, 75 (1997) arXiv:hep-th/9702103.

[24] A. Ceresole, R. D'Auria and S. Ferrara, "The Symplectic Structure of N=2 Supergravity and its Central Extension," Nucl. Phys. Proc. Suppl. 46, 67 (1996) arXiv:hep-th/9509160]. 
[25] M. J. Duff, "String triality, black hole entropy and Cayley's hyperdeterminant," arXiv:hep-th/0601134.

[26] M. J. Duff, J. T. Liu and J. Rahmfeld, "Four-dimensional string-string-string triality," Nucl. Phys. B 459, 125 (1996) hep-th/9508094.

[27] B. Bates and F. Denef, "Exact solutions for supersymmetric stationary black hole composites," arXiv:hep-th/0304094.

[28] F. Denef, "On the correspondence between D-branes and stationary supergravity solutions of type II Calabi-Yau compactifications," arXiv:hep-th/0010222;

[29] F. Denef, "Supergravity flows and D-brane stability," JHEP 0008, 050 (2000) arXiv:hep-th/0005049. 